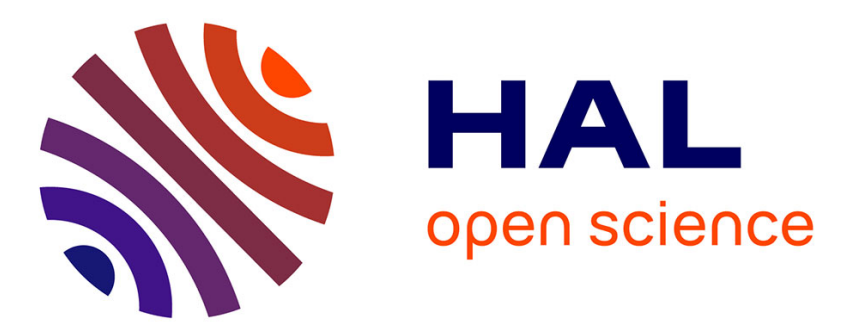

\title{
A robust hypothesis test for the sensitive detection of constant speed radiation moving sources
}

\author{
Jonathan Dumazert, Romain Coulon, Vladimir Kondrasovs, Karim \\ Boudergui, Yoann Moline, Guillaume Sannié, Jean-François Gameiro, \\ Stéphane Normand, Laurence Méchin
}

\section{To cite this version:}

Jonathan Dumazert, Romain Coulon, Vladimir Kondrasovs, Karim Boudergui, Yoann Moline, et al.. A robust hypothesis test for the sensitive detection of constant speed radiation moving sources. Nuclear Instruments and Methods in Physics Research Section A: Accelerators, Spectrometers, Detectors and Associated Equipment, 2015, 795, pp.335-342. 10.1016/j.nima.2015.06.016 . hal-01194701

\section{HAL Id: hal-01194701 \\ https://hal.science/hal-01194701}

Submitted on 7 Sep 2015

HAL is a multi-disciplinary open access archive for the deposit and dissemination of scientific research documents, whether they are published or not. The documents may come from teaching and research institutions in France or abroad, or from public or private research centers.
L'archive ouverte pluridisciplinaire $\mathbf{H A L}$, est destinée au dépôt et à la diffusion de documents scientifiques de niveau recherche, publiés ou non, émanant des établissements d'enseignement et de recherche français ou étrangers, des laboratoires publics ou privés. 


\title{
A robust hypothesis test for the sensitive detection of constant speed radiation moving sources
}

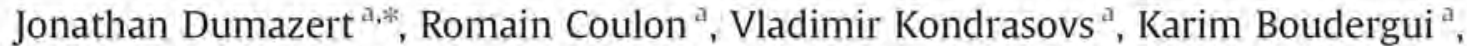 \\ Yoann Moline ", Guillaume Sannié ${ }^{a}$, Jordan Gameiro ${ }^{a}$, Stéphane Normand ${ }^{a}$, \\ Laurence Méchin ${ }^{\mathrm{b}}$ \\ CEA, LIST, Laboratoire Capteurs Architectures Electroniques, 91191 Gif-sur-Yvette, France \\ b CNRS, UCBN. Groupe de Recherche en Informatique, Image. Automatique et Instrumentation de Caen, 14050 Caen, France
}

\begin{abstract}
A B S T R A C T
Radiation Portal Monitors are deployed in linear networks to detect radiological material in motion. As a complement to single and multichannel detection algorithms, inefficient under too low signal-to-noise ratios, temporal correlation algorithms have been introduced. Test hypothesis methods based on empirically estimated mean and variance of the signals delivered by the different channels have shown significant gain in terms of a tradeoff between detection sensitivity and false alarm probability. This paper discloses the concept of a new hypothesis test for temporal correlation detection methods, taking advantage of the Poisson nature of the registered counting signals, and establishes a benchmark between this test and its empirical counterpart. The simulation study validates that in the four relevant configurations of a pedestrian source carrier under respectively high and low count rate radioactive backgrounds, and a vehicle source carrier under the same respectively high and low count rate radioactive backgrounds, the newly introduced hypothesis test ensures a significantly improved compromise between sensitivity and false alarm. It also guarantees that the optimal coverage factor for this compromise remains stable regardless of signal-to-noise ratio variations between 2 and 0.8 , therefore allowing the final user to parametrize the test with the sole prior knowledge of background amplitude.
\end{abstract}

\section{Introduction and related work}

The detection of radiological material in motion forms a burning issue in addressing CBRN threats [1]. Whether the potential carrier may be a pedestrian, a train or a vehicle, Radiation Portal Monitors (RPM) are deployed so that the radiation sensor inserted into the device is placed as close as possible to the radioactive source path. Classical industrial detection systems for moving sources perform the detection by mere signal amplitude triggering on a recording channel interfaced with the sensor. The alarm of the detector is triggered as soon as the relevant signal rises over a given threshold, which is set with regards to the amplitude of the background activity as well as to some assumptions formulated based on the laws followed by the different signals. For the detection to be efficient when the carrier is in motion, a large volume sensor is supplied, typically a gas or plastic scintillator for scalability and cost-effectiveness reasons (2.3). To obtain significant gains in the richness of the information provided by such a single RPM, ad hoc strategies have been developed. Guillot et al. have, for instance, described and patented [4] a method for the detection and identification of moving sources using a spectrometry device, which lies outside the scope of the present paper, focused on pure detection applications. To increase the sensitivity of the detection. especially critical under low signal-to-noise ratios (SNR, for which we hereby use the classical definition of the source signal count divided by the square root of the background fundamental count), energy windowing strategies have been introduced. Robinson et al. [5] compare at each given time the observed spectrum to adequately chosen energy windows over a background previously acquired at the RPM level. To maximize the gain in sensitivity, Vilim et al. [6] select the energy ranges providing the highest SNR values. It then follows that such methods are highly dependent on the energy resolution of the provided spectrometric information. Hence the quest for alternative methods continues for utilizing large-scale plastic scintillators exhibiting high detection efficiencies but mediocre energy resolutions.

A powerful strategy to address sensitivity issues when dealing with challenging SNR moving sources lies within the deployment of not only one RPM, but a network of such sensors. While independent multichannel triggering remains available for such a network. 
the measurement then being computed by the logical summation of all channel detection answers, some valuable information may be extracted by analyzing the delayed temporal evolution of the signals recorded in front of the respective RPM. Such networks, of variable geometries and dimensions, are particularly praised for source localization applications, in which they typically appear twodimensional (2-D) and large-scale. Dedicated algorithms have then been elaborated to track the source trajectory without prior knowledge, as carried out with Markov Chains calculations and Bayesian methods by Brennan et al. [7] or triangulation by Chin et al. [8]. Such highly iterative and finely optimized methods are poorly suited for typical CBRN issues dealing with straight trajectories of pedestrian or vehicle source carriers under real time, immediate response constraint. Alternatively, faster algorithms have been proposed: Nemzek et al. [9] describe a method based on the combination of different channels and final comparison to the signal of a single chosen RPM. Stephens et al. [10] additionally justify a preliminary probabilistic triggering on the acquisition channels. Both these methods nevertheless require that the speed of the source carrier be known with a certain degree of precision, which in real life situations is difficult to achieve and may thus constitute an excessive constraint for the detection system conceiver. Sundaresan et al. [11] have, on the other hand, set up a measurement based on a priori independent multichannel detections which are all correlated a posteriori to lower the false alarm probability. Such an approach, as firstly based on a single channel variation monitoring, does not allow for any sensitivity improvement of the system, so that the false alarm reduction does not reveal itself sufficient to operate the detector under challengingly low SNR. Rao et al. have additionally established in a recent paper [12] that a network of Nal sensors associated with a particle filter method allows the detection of a cesium-137 moving source with shorter delays and over a wider spatial range when combining the data acquired by several networked detectors.

Coulon et al. have described and patented $[13,14]$ an alternative exploitation of the compared temporal information contained in the different recording channel memories. As the source carrier, supposedly following a linear or quasi-linear trajectory, successively passes in front of each of the RPM, displayed in a network, it induces for a short time an increase in the signal level. Such an increase, in the cases of challenging SNR, may be impossible to detect in front of the first RPM among the inherent level of fluctuations induced by the Poisson statistical nature of the measured radiation background. Nevertheless, supposing that the carrier moves at constant speed or quasi-constant speed along the network (which forms a more versatile assumption than the one of a precisely determined constant speed and a reasonable one for a pedestrian, a car or a train before its final deceleration), an echo of the undetected additional signal may be found on every other channel and, as the speed is constant, these successive appearances are periodic. It is therefore possible to search for a temporal delay, on the form of a multiple value of the fundamental sampling time step; which will, to the maximum possible extent, superimpose the successive echoes of the undetected signal increases and exploit the multiplied values of these echoes as the significant variation to be detected, by a specified amplitude triggering, among the inherent fluctuations of a computed temporal multiplication vector. Coulon et al. have calibrated a hypothesis test for moving source detection without any assumption made on the expected nature and intensity of such a product vector, making use of the empirical estimates for the mean and standard deviation of the vector. Their approach has proven itself efficient for a relatively high speed $\left(7 \mathrm{~m} . \mathrm{s}^{-1}\right)$ low count rate $(12 \mathrm{cps})$ source among a challenging count rate background $(20 \mathrm{cps})$. A preliminary set of simulations has additionally corroborated the choice of Coulon et al. to search for the echo of the source passage in the product rather than in the summation of the different channel recordings, the higher amplification of the background then being advantageously balanced by a vast gain in the amplitude of the useful signal. The authors of the present paper propose to investigate an improved version of the detection test introduced by Coulon et al. first describing the underlying statistics governing the temporal product vector used for the test, and then parametrizing the said test by a novel expectation and widening as computed under the assumption of a product of Poisson laws.

\section{Mathematical formalism}

Let us consider the signals registered on the two RPM of a given linear network to be generated by two real and independent underlying random variables $S_{1}$ and $S_{2}$ with Poisson density laws respectively parametrized by $\lambda_{1}$ and $\lambda_{2}$. Providing $\lambda_{1}$ and $\lambda_{2}$ are high enough, both distributions may be approached by the normal distributions $\mathcal{N}\left(\lambda_{1}, \lambda_{1}\right)$ and $\mathcal{N}\left(\lambda_{2}, \lambda_{2}\right)$. We consider the aleatory variable $Y=S_{1} \cdot S_{2}$ formed by the product of both previous ones.

\subsection{Calculation of the expected value and variance of $Y$}

The expected value $E[Y]$ of the random variable formed by the product of two real and independent variables is given by the product of both expected values $E\left[S_{1}\right]$ and $E\left[S_{2}\right]$ of the considered variables

$E[Y]=E\left[S_{1}+S_{2}\right]=E\left[S_{1}\right] \cdot E\left[S_{2}\right]=\lambda_{1} \cdot \lambda_{2}$

The variance $V(Y)$ of $Y$ is a function of both variances $V\left(S_{1}\right)$ and $V\left(S_{2}\right)$ of the multiplied variables, as well as of their expected values $E\left[S_{1}\right]$ and $E\left[S_{2}\right]$

$$
\begin{aligned}
& V(Y)=V\left(S_{1}\right) \cdot V\left(S_{2}\right)+V\left(S_{1}\right) \cdot\left(E\left[S_{2}\right]\right)^{2}+V\left(S_{2}\right) \cdot\left(E\left[S_{1}\right]\right)^{2} \\
& V(Y)=\lambda_{1} \cdot \lambda_{2} \cdot\left(1+\lambda_{1}+\lambda_{2}\right)
\end{aligned}
$$

\subsection{Generalization to the product of $n$ variables}

The previous formalism may be generalized to the case of a linear network displaying any number $n$ of RPM. Let us then consider $n$ real and independent random variables $S_{1}, S_{2} \ldots S_{n}$ with Poisson density laws respectively parametrized by $\lambda_{1}, \lambda_{2}, \ldots \lambda_{n}$, which are respectively approached by the normal law distributions $\mathcal{N}\left(\lambda_{1}, \lambda_{1}\right), \mathcal{N}\left(\lambda_{2}, \lambda_{2}\right) \ldots \mathcal{N}\left(\lambda_{n}, \lambda_{n}\right)$. We consider the random variable $Y=S_{1} \cdot S_{2} \ldots S_{n}$ formed by the product of the $n$ previous ones.

The expected value $E[Y]$ of the variable defined as the product of $n$ real and independent variables is given by the product of the expected values $E\left[S_{1}\right], E\left[S_{2}\right] \ldots E\left[S_{n}\right]$ of the considered variables

$E[Y]=E\left[S_{1}\right]-E\left[S_{2}\right] \ldots E\left[S_{n}\right]=\lambda_{1}+\lambda_{2} \ldots \lambda_{n}=\prod_{i=1}^{n} \lambda_{i}$

The calculation of the variance $V(Y)$ of $Y$ is carried out by applying recursively the result of Section 2.1

$$
\begin{aligned}
V(Y)= & V\left(S_{1} \cdot S_{2} \ldots S_{n-1}\right) \cdot V\left(S_{n}\right)+V\left(S_{1} \cdot S_{2} \ldots S_{n-1}\right) \cdot\left(E\left[S_{n}\right]\right)^{2} \\
& +V\left(S_{n}\right) \cdot\left(E\left[S_{1} \cdot S_{2} \ldots S_{n-1}\right]\right)^{2} \\
V(Y)= & V\left(S_{1} \cdot S_{2} \ldots S_{n-1}\right) \cdot\left(\lambda_{n}+\lambda_{n}^{2}\right)+\lambda_{n} \cdot\left(\prod_{i=1}^{n-1} \lambda_{i}\right)^{2}
\end{aligned}
$$

The same treatment is then applied to the variables $S_{1} \cdot S_{2} \ldots S_{n-1}$, then $S_{1} \cdot S_{2} \ldots S_{n-2} \ldots$ lowering the number of factors after every iteration until only 2 remain and we are therefore brought back to the case dealt with in Section 2.1 .

\section{Method}

This section divulges the steps followed by the simulation code (simulation of the recorded radiation signal, data storage, calculation 
of the product vector, hypothesis tests) to assess the respective performances of different algorithms for the detection of a radiation moving source passing in front of $n$ equidistant RPM arrayed in a linear network.

\subsection{Simulation of the radiation signal, time depth and memory buffer management}

Let the number of RPM displayed be labeled $n$, the distance of closest approach to an RPM for the source carrier $d_{1}$, the distance between two adjacent detectors $d_{2}$, the carrier speed $v$, which is supposed to be constant or negligibly varying for the type of applications we are aiming at, the background radiation count rate $\lambda_{b}$ and the source count rate in front of one detector $\lambda_{s}$. The instantaneous count rate $\lambda_{j}\left[t_{i}\right]$ which is expected to be registered at the ith integration time step by the $j$ th RPM detector, described as a function of the time-dependent source carrier position $x\left[t_{i}\right]$, is provided by (5). The calculation has been carried out under the approximation that the source and the detector may be modeled by their centers of mass and therefore that the principle of the ratio of solid angles applies.

$\forall j \in 1 ; n, \forall i \in 1 ; M, \lambda_{j}\left[t_{i}\right]=\frac{\lambda_{5} \cdot d_{1}^{2}}{d_{1}^{2}+\left(x\left[t_{i}\right]-x_{j}\right)^{2}}+\lambda_{b}$

The recording time step is designated as $\Delta t=t_{i+1}-t_{i}$. Given the introduced notations, the SNR is evaluated as stated in (6)

$\operatorname{SNR}=\frac{\lambda_{s} \cdot \Delta t}{\sqrt{\lambda_{b} \cdot \Delta t}}=\frac{\lambda_{s} \cdot \sqrt{\Delta t}}{\sqrt{\lambda_{b}}}$

For each of the $j$ RPM channel, a time-varying count is generated following an inhomogeneous Poisson process modeled with the use of the "Poissrnd" $\varnothing$ pseudo-random function of Matlab parametrized by the time-varying value of the underlying count rate $\lambda_{j}$. A third-order moving averaging $M A(., 3)$ is applied to the count rate vector previous to any further operation, so that the expectation for the recorded signal $S_{j}\left[t_{i}\right]$ at the ith integration time step by the $j$ th RPM detector is provided by (7).

$\forall j \in 1 ; n, \forall i \in 1 ; M, S_{j}\left[t_{i}\right]=M A\left(\wp_{0}\left(\lambda_{j}\left[t_{i}\right]-\Delta t\right), 3\right)$

For every $j$ channel and every discrete time step $t_{i}$ a new value is recorded in a signal matrix $\left(S_{i, j}=S_{j}\left[t_{i}\right]\right)_{i=1} M_{j=1, n}$, where $M$ represents the time depth of the recording and the memory buffer size allocated at every independent detector level.

\section{3,2. Calculation of the temporal product vector}

Both the hypothesis test divulged by Coulon et al. which is assumption-free on the distributions followed by the radiation signals, and the one we introduce in this paper, based on a Poisson law assumption, make use of the same temporal product vector $\left(R_{k}\right)_{k=1 \ldots N_{k}}$ of length $N_{R}=\left[\frac{n}{M}\right]$. The $k$ parameter quantifies the shift between the first RPM data vector $S_{i, 1}$ and the data vectors obtained with the following RPM: $S_{i, 2}$ will be shifted of $k, S_{i, 2}$ of $2 k, S_{i, 3}$ of $2^{2} k$ and so on. For every discrete value of $k$ between 1 and $N_{k}$, the value stored in $\left(R_{k}\right)_{k=1 . N_{\mu}}$ is the maximum of the vector obtained by the two-by-two product of the shifted terms in the respective data sets. The exploitation of the temporal product vector for the detection will be detailed in the following subsection. The $k$ th term of the product vector is calculated as described in ( 8 ) and (9) for $n=2$ and $n=4$ respectively:

$\forall k \in 1: N_{k}, R_{k}=\max _{i \in 1: M-k+1}\left(S_{i, 1} \cdot S_{i+k-1,2}\right)$ $\forall k \in 1 ; N_{R}, R_{k}=\max _{i \in 1: M-4(k-1)}\left(S_{i, 1} \cdot S_{i+k-1,2} \cdot S_{i+2(k-1), 3} \cdot S_{i+4(k-1), 4}\right)$

\subsection{Hypothesis tests for the detection}

Any of the benchmarked hypothesis tests for the detection of a radiative source is based on the comparison between a registered signal and a decision threshold set with regards to both the expected amplitude and the distribution of the signal around this expected amplitude.

The simplest hypothesis test only makes use of one RPM, say the first in the network. If we define $\mathrm{H}_{0}$ as the assertion "no source is passing in front of the RPM network" and $\mathrm{H}_{1}$ as the assertion "a source is passing in front of the RPM network", the acceptance of $\mathrm{H}_{1}$ is parametrized by a coverage factor $K$ according to (10) so that if

$\max _{i \in 1: M}\left(S_{i, 1}\right)>\overline{S_{-1}}+K \cdot \sigma\left(S_{-1}\right)$

then the alarm is triggered and a source is detected. The estimator of the expected value $\overline{S_{-1}}$ and the estimator of the standard deviation $\sigma\left(S_{11}\right)$ over the count rate vector are respectively calculated with the formulae displayed in (11) and (12):

$\overline{S_{, 1}}=\frac{1}{M} \sum_{i=1}^{M} S_{i, 1}$

$\sigma\left(S_{. .1}\right)=\sqrt{\frac{1}{M-1} \sum_{i=1}^{M}\left(S_{i, 1}-\overline{S_{.1}}\right)^{2}}$

The factor $K$ is investigated with regards to the best compromise between false alarm probability $\left(P_{F A}=P\left(H_{1} \mid H_{0}\right)\right.$ by definition), which corresponds to the acceptance of $\mathrm{H}_{1}$ in the absence of a moving source $\left(\lambda_{s}=0\right)$ and the detection probability $\left(P_{D}=P\left(H_{1} \mid H_{1}\right)\right.$ by definition), which represents the acceptance of $\mathrm{H}_{1}$ in the presence of a source. This compromise may be figured by Receiver Operating Characteristic [15] (ROC) plotting the evolution of $P_{D}$ as a function $P_{F A}$ or, if no critical $P_{F A}$ has been previously defined for the benchmark, by the evolution of the intuitive factor of merit labeled FOM and trivially calculated following (13):

$F O M=P_{D}-P_{F A}$

Under unfavorable SNR, the canonic strategy, which consists in triggering the alarm whenever the hypothesis test defined in (9), for a preset value of $K$, results in the acceptance at the level of any of the $n$ RPM displayed in the network (OR logical function), results in unacceptably low FOM values, as Coulon et al. have already demonstrated. Hence the development of alternative hypothesis tests based on the superimposition of the signals registered on all channels, as built in the temporal product vectors.

The hypothesis test we wish to benchmark the new algorithm disclosed in this paper to adds one acceptance condition for $\mathrm{H}_{1}$ in the OR logical function over the $n$ independent tests introduced supra, which is stipulated in (14):

$\max _{k \in 1: N_{k}}\left(R_{k}\right)>\bar{R}+K \cdot \sigma(R)$

The estimator of the expected value $\bar{R}$ and the estimator of the standard deviation $\sigma(R)$ over the temporal product vector are respectively calculated with the formulae displayed in (15) and (16):

$\bar{R}=\frac{1}{N_{R}} \sum_{k=1}^{M} R_{k}$

$\sigma(R)=\sqrt{\frac{1}{N_{R}-1} \sum_{i=1}^{N_{k}}\left(R_{k}-\bar{R}\right)^{2}}$ 
The factor $K$ is investigated with regards to the optimal tradeoff represented by the maximization of FOM.

The concept of the alternative algorithm introduced in this paper lies within the replacement of $\bar{R}$ and $\sigma(R)$ by estimations of the expected value and variance of the correlation vector based on the theoretical derivation of Section 2, hence making use of the knowledge of the Poisson nature of the underlying laws to the registered count rates. In this alternative hypothesis test, the additional acceptance condition for $\mathrm{H}_{1}$ in the OR logical function over the $n$ independent tests writes as described in (17) and (18) for $n=2$ and $n=4$ respectively, deduced from (1)-(4):

$\max _{k \in 1: N_{k}}\left(R_{k}\right)>\overline{S_{.1}} \cdot \overline{S_{.2}}+K \cdot \sqrt{S_{.1} \cdot \overline{S_{.2}} \cdot\left(1+\overline{S_{.1}}+\overline{S_{.2}}\right)}$

$\max _{k \in 1 ; N_{k}}\left(R_{k}\right)>\prod_{j=1}^{4} \overline{S_{j}}+K \cdot \sqrt{\Pi \cdot\left(\overline{S_{. .4}}+\overline{S_{.4}} 2\right)+\overline{S_{. .4}} \cdot\left(\prod_{j=1}^{3} \overline{S_{. j}}\right)^{2}}$

where the factor $\Pi$ is calculated as made explicit in (19)

$\Pi=\overline{S_{.1}} \cdot \overline{S_{.2}} \cdot\left(1+\overline{S_{.1}}+\overline{S_{.2}}\right) \cdot\left(\overline{S_{.3}}+\overline{S_{33}}\right)+\overline{S_{33}} \cdot\left(\overline{S_{.1}} \cdot \overline{S_{.2}}\right)^{2}$

For every $j$ channel, the expected count value $S_{j, j}$ is evaluated according to $(20)$

$\forall j \in 1 ; n, \overline{S_{4,}}=\frac{1}{M} \sum_{i=1}^{M} S_{i, j}$

The factor $K$ is once again varied until the maximum value for FOM is found.

\section{Results}

\subsection{Assessment scheme}

To access the characteristic representing the evolution of FOM as a function of the coverage factor $K$, in the four previously introduced configurations of implementation, we generate for every of these situations and every $S N R$ value considered in them, a number $N=1000$ times the activity $\lambda_{s}$ we wish to detect and the same $N=1000$ times a null activity $\lambda_{s}=0$ to evaluate the false alarm probability in the described background and detection scheme. If we denote $N_{D}$ is the number of detection cases in the first simulation batch (non-null activity) and $N_{F A}$ is the number of detection cases in the second batch (null activity), then the quantities $P_{D}$ and $P_{F A}$ are defined in Section 3.3, with their respective standard deviations $\sigma\left(P_{D}\right)$ and $\sigma\left(P_{F A}\right)$ (quantifying the repeatability uncertainty lying upon them), are estimated as shown in (21) -(24).

$P_{D}=\frac{N_{D}}{N}$

$\sigma\left(P_{D}\right)=\sqrt{\frac{1}{N} \cdot P_{D} \cdot\left(1-P_{D}\right)}$

$P_{F A}=\frac{N_{F A}}{N}$

$\sigma\left(P_{F A}\right)=\sqrt{\frac{1}{N} \cdot P_{F A} \cdot\left(1-P_{F A}\right)}$

Ultimately the performance indicator FOM is evaluated as the difference between $P_{D}$ and $P_{F A}$, and its associated uncertainty $\sigma(F O M)$ is computed as exhibited on (25).

$\sigma(F O M)=\sqrt{\sigma^{2}\left(P_{D}\right)+\sigma^{2}\left(P_{F A}\right)}$
The number of iterations $N=1000$ has been set so that, for all the results presented in Section 5 , the overall uncertainty $\sigma(F O M)$ never exceeds the value of $0.02(2 \%)$. The recording time step $\Delta t=t_{i+1}-t_{i}$ and the buffer size $M$, which are both fixed parameters for all simulations whose results are divulged in the four following subsections, are respectively set to $0.1 \mathrm{~s}$ and 100 , which are compatible with the constraint of a quasi-continuous monitoring of radioactivity.

\subsection{Pedestrian carrier with high and low count rate background}

The first real-life challenging situation we intend to simulate is that of a RPM network displayed to detect a radioactive source carried by a pedestrian with a usually high count rate background (for instance nearby a facility dedicated to the storage and handling of sources for research purposes, which is the typical background encountered during the first experimental assessment of a new detection sensor and/or algorithm or an environment of critical interest for radioprotection issues, and with large-scale RPM sensors). The value chosen for the background count rate $\lambda_{b}=2000 \mathrm{cps}$. A realistic value for the speed of the pedestrian, who is to walk alongside the detector network at a distance $d_{1}=1 \mathrm{~m}$, is set at $v=5 \mathrm{~km} \mathrm{~h}^{-1}$. As this speed is low, so is the spacing between adjacent RPM in the line, which is put equal to $d_{2}=2 \mathrm{~m}$. The FOM value is computed and displayed on Figs. 1-4 for four increasing challenging values of signal-to-noise ratios: $S N R=2, S N R=1.5$, $S N R=1$ and $S N R=0.8$. On every figure, we have superimposed, with their respective optimization ranges with regards to $K$, the values of FOM obtained for an independent detection on the first RPM (M1FOM), for a detection using the product vector and empirical mean and variance as done by Coulon et al. with a linear network of $n=2$ (R2FOM) and $n=4$ (R4FOM) RPM respectively, and for a detection using the product vector and a Poisson hypothesis on the mean and variances as disclosed in this paper with a linear network of $n=2$ (PG2FOM) and $n=4$ (PG4FOM) RPM respectively.

The second real-life challenging situation we intend to simulate is that of a small or moderate-size sensor network (typically 1$10 \mathrm{~cm}$ height and diameter) displayed to detect a radioactive source once again carried by a pedestrian, though this time with a usually very low count rate background (as in most typical CBRN applications). The value chosen for the background count rate $\lambda_{b}=5 \mathrm{cps}$ is additionally specified as extremely low so that the present study may cover the range defined by Coulon et al. $(20 \mathrm{cps})$. The values $d_{1}=1 \mathrm{~m}, v=5 \mathrm{~km} \mathrm{~h}^{-1}$ and $d_{2}=2 \mathrm{~m}$, well suited for the monitoring of a flow of pedestrians, remain unchanged from the previous settings. Figs. 5-8 display the same characteristics of FOM as a function of $K$ as those disclosed in high count rate situations, where the activity $\lambda_{s}$ is set to simulate the same four values of SNR and where the same five detection tests are assessed.

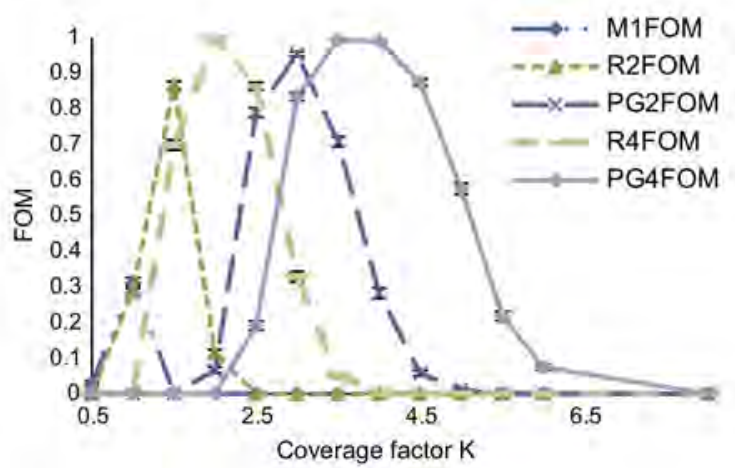

Fig. 1. Pedestrian $\left(\lambda_{b}=2000 \mathrm{cps}: S N R=2\right)$ 


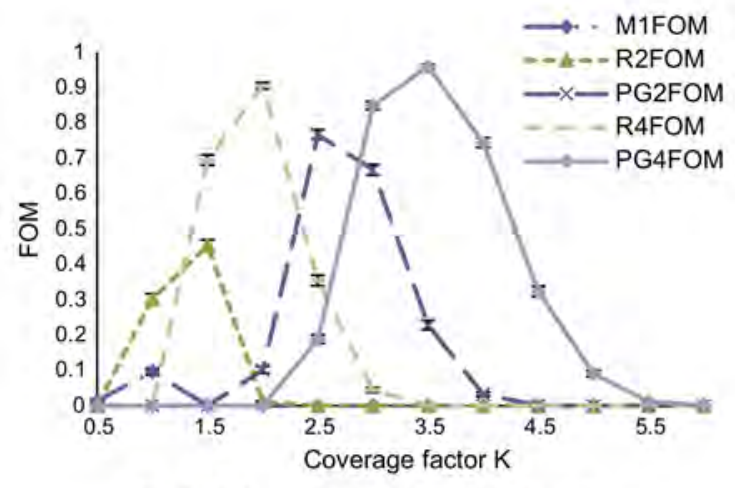

Fig. 2. Pedestrian $\left(\lambda_{b}=2000 \mathrm{cps} ; S N R=1.5\right)$.

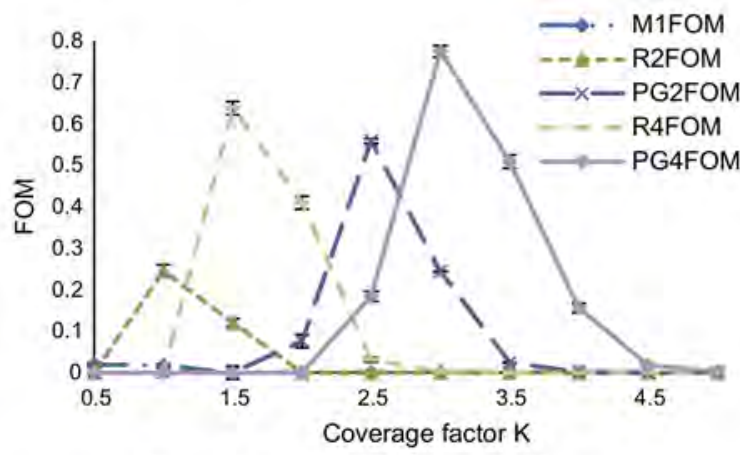

Fig. 3. Pedestrian $\left(\lambda_{b}=2000 \mathrm{cps} ; S N R=1\right)$.

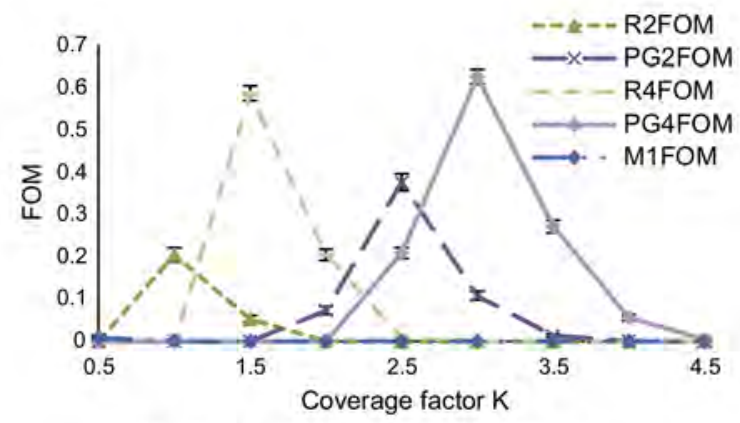

Fig. 4. Pedestrian $\left(\lambda_{b}=2000 \mathrm{cps} ; S N R=0,8\right)$.

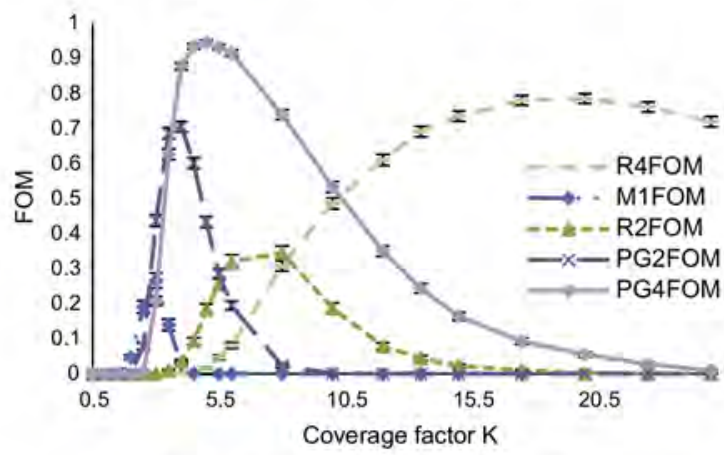

Fig. 5. Pedestrian $\left(\hat{\lambda}_{b}=5 \mathrm{cps} ; S N R=2\right)$.

\subsection{Vehicle carrier with high and low count rate background}

The third real-life challenging situation we wish to simulate is that of a RPM network displayed to detect a radioactive source

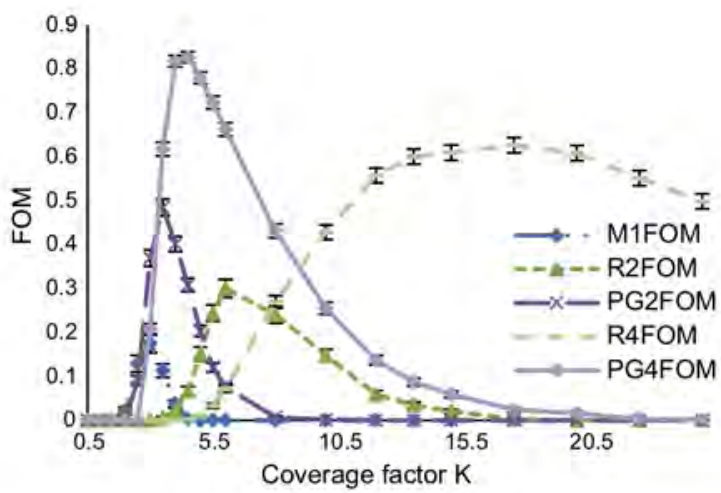

Fig. 6. Pedestrian $\left(\lambda_{b}=5 \mathrm{cps} ; S N R=1.5\right)$.

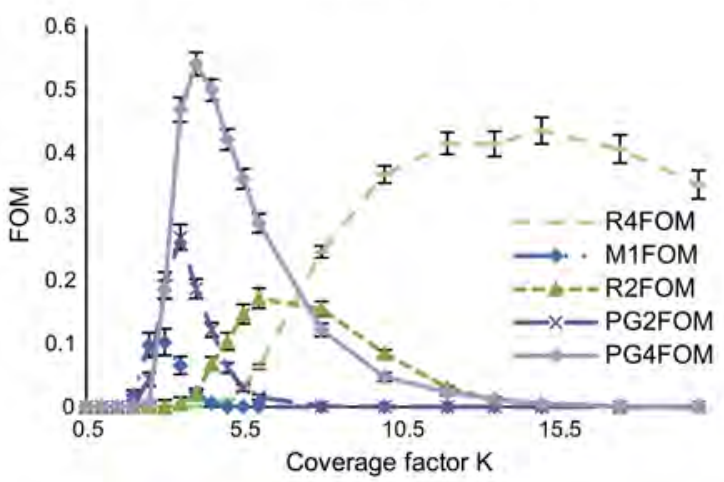

Fig. 7. Pedestrian $\left(\lambda_{b}=5 \mathrm{cps} ; S N R=1\right)$.

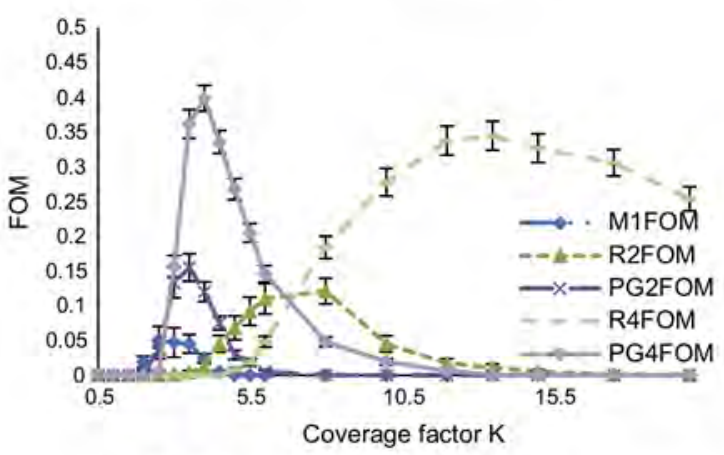

Fig. 8. Pedestrian $\left(\lambda_{b}=5 \mathrm{cps} ; 5 N R=0.8\right)$.

carried by a vehicle, whether that may be a car entering a restricted access area or a train entering a station. We keep for these simulations the same value for the high background count rate $\lambda_{b}=2000 \mathrm{cps}$ and associated activities $\lambda_{s}$ to provide the four $S N R$ values lying between 2 and 0.8 . On the contrary, a different set of parameters is selected to represent both the carrier and the RPM linear network, which meets practical requirements: $d_{1}=3 \mathrm{~m}, v=15 \mathrm{~km} \mathrm{~h}^{-1}$ and $d_{2}=15 \mathrm{~m}$. Figs. 9-12 display the FOM characteristics for all simulated SNR and all five benchmarked hypothesis tests.

The fourth and last real-life challenging situation we wish to simulate is that of a RPM network displayed to detect a radioactive source carried by a vehicle in a low count rate background, set equal to $\lambda_{b}=5 \mathrm{cps}$ as in Section 5. The parameters $d_{1}=3 \mathrm{~m}$, $v=15 \mathrm{~km} \mathrm{~h}^{-1}$ and $d_{2}=15 \mathrm{~m}$ remain unchanged. Figs. 13-16 eventually disclose the FOM characteristics for all four simulated SNR and all five benchmarked hypothesis tests. 


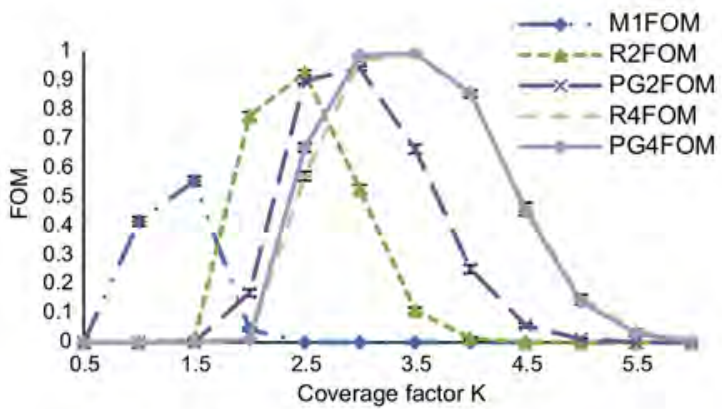

Fig. 9. Vehicle $\left(\lambda_{b}=2000 \mathrm{cps} ; \mathrm{SNR}=2\right)$.

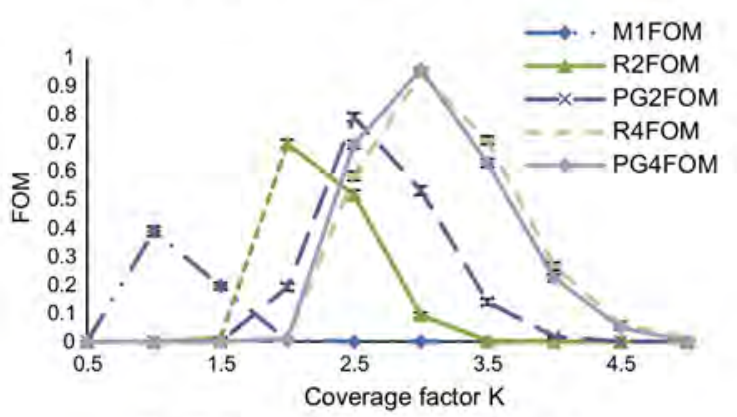

Fig. 10. Vehicle $\left(\lambda_{b}=2000 \mathrm{cps} ; S N R=1.5\right)$.

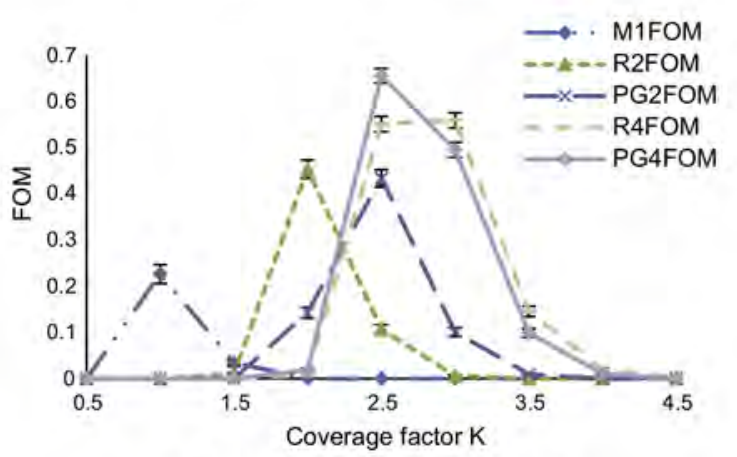

Fig. 11. Vehicle $\left(\lambda_{b}=2000 \mathrm{cps}\right.$; $\left.S N R=1\right)$.

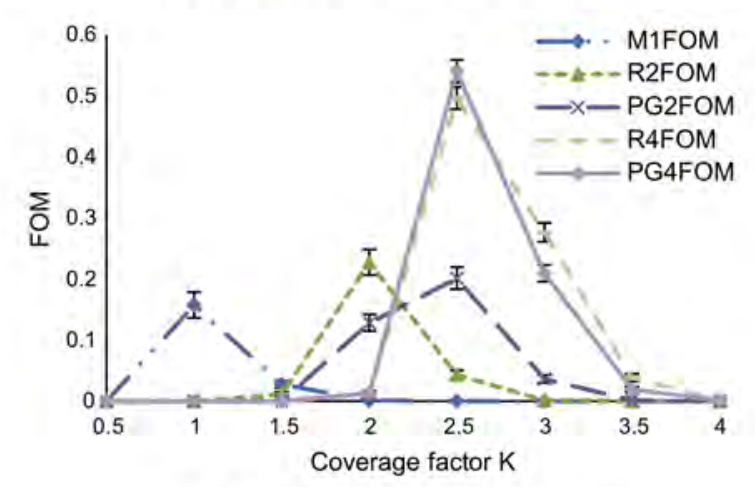

Fig. 12. Vehicle $\left(\lambda_{b}=2000 \mathrm{cps}: S N R=0.8\right)$.

\section{Discussion}

The characteristics displayed in Figs. 1-16 do not include an independent multichannel test over $n=2$ and $n=4$ RPM, for its performance usually does not significantly differ from the ones of M1FOM, except for $S N R=2$ which represents the least challenging case and thus the least relevant for the present comparative study.

The first conclusions drawn from the simulation results converge to corroborate the claims made in Coulon et al. about the

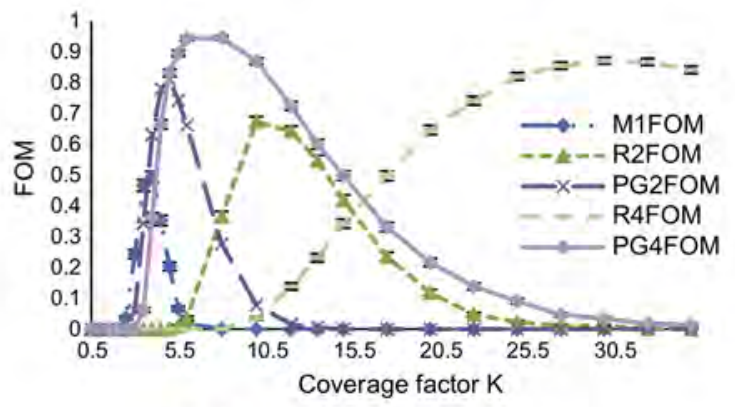

Fig. 13. Vehicle $\left(\lambda_{b}=5 \mathrm{cps} ; S N R=2\right)$,

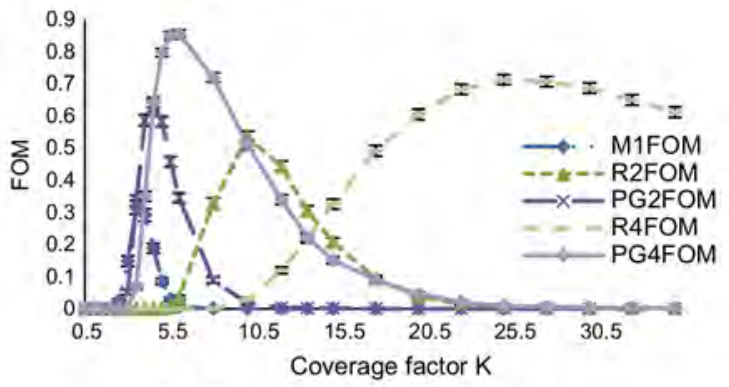

Fig. 14. Vehicle $\left(\lambda_{b}=5 \mathrm{cps} ; S N R=1.5\right)$.

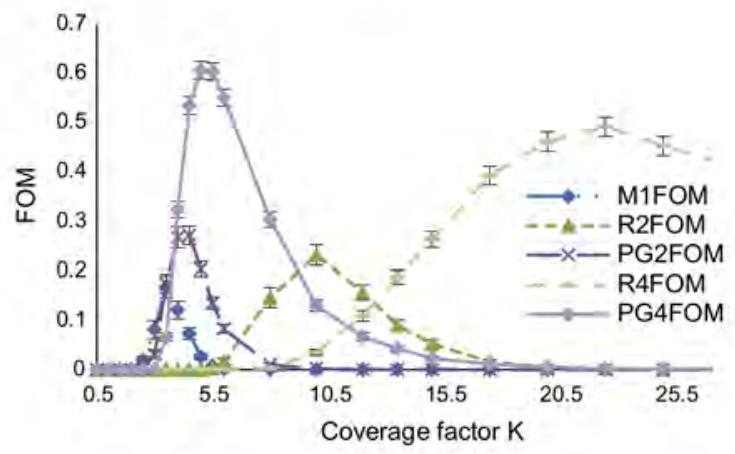

Fig. 15. Vehicle $\left(\lambda_{b}=5\right.$ cps; $\left.S N R=1\right)$.

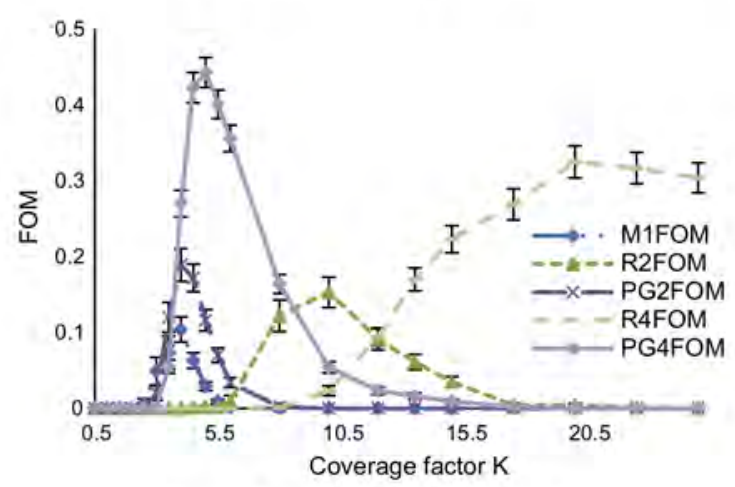

Fig. 16. Vehicle $\left(\lambda_{b}=5 \mathrm{cps} ; S N R=0.8\right)$.

general merits of temporal multiplication, regardless of the chosen hypothesis test. In all four configurations (pedestrian with $\lambda_{b}=2000 \mathrm{cps}$, pedestrian with $\lambda_{b}=5 \mathrm{cps}$, vehicle with $\lambda_{b}=2000 \mathrm{cps}$, vehicle with $\lambda_{b}=5 \mathrm{cps}$ ), the exploitation of the echo, identified with the maximum over a range of shift parameters $k$ of the temporal product vector $\left(R_{k}\right)_{k=1 \ldots N_{k}}$ as utilized in (14), (17) and (18), allows a drastic improvement of the FOM value under low SNR conditions, almost systematically reaching a $10-20 \%$ increase from M1FOM to R2FOM or PG2FOM, and $30-40 \%$ increases from 
M1FOM to R4FOM to PG2FOM. Our results additionally confirm that, for both hypothesis tests making use of the temporal product vector, the performances increase with the number of RPM displayed in the network. We have therefore widened the promising conclusions of Coulon et al. from a particular configuration suiting a vehicle carrier in a relatively low count rate background to a larger spectrum of concrete applications.

The core of this paper lies within the two-by-two comparisons of the characteristics of R2FOM and PG2FOM one the hand, and R4FOM and PG4FOM on the other hand, as PG2FOM and PG4FOM result from the original hypothesis test hereby disclosed and described in (17)-(20). The most obvious observation from Figs. $1-16$ is that the maximum of the PG2FOM is constantly superior to the maximum of R2FOM, and similarly that the maximum of PG4FOM exceeds the maximum of R4FOM. The discrepancy between PG4FOM and R4FOM is particularly noticeable under $\lambda_{b}=5 \mathrm{cps}$ background. As any of the chosen tests for the detection of moving source should be set without prior knowledge of the SNR, the stability of the value for the coverage factor $K$ providing the best FOM is another critical figure of merit. A superficial look at the characteristics reveals that the optimization range for $K$ differs greatly for the test making use of the empirical mean and variance and our test under Poisson assumption, except in the case of a vehicle carrier with $\lambda_{b}=2000 \mathrm{cps}$. The distributions of R2FOM and R4FOM exhibit extremely smooth profiles around their maximum with low count rate background, as it had already been reported by Coulon et al. which guarantees a high degree of robustness of the test for a value of $K$ chosen in the most important FOM values area and a SNR varying between 2 and 0.8 . The characteristics of PG2FOM and PG4FOM are sharper around their maximum value, but still ensure a robust detection setting for varying SNR. For instance, Figs. $1-4$ show that an initial setting of $K=3$ guarantees PG4FOM values of respectively $0.83,0.85,0.78$ and 0.63 for $S N R=2, S N R=1.5, S N R=1$, and $S N R=0.8$, which are both satisfactory in absolute terms given the unfavorable SNR under which they are obtained and also superior to the concurrent performances disclosed by the R4FOM characteristics over the same SNR range. Similarly, Figs. 13-16 show that an initial setting of $K=5.5$ guarantees PG4FOM values of respectively $0.90,0.85$ and 0.61 for $S N R=2, S N R=1.5$ and $S N R=1$ representative of the best performance attained over the entire coverage factor span with the test introduced in this paper and overcoming the maximum value of the R4FOM characteristic by 3-14\% depending on the SNR.

\section{Conclusions and perspectives}

The simulation study in various realistic radioactive backgrounds and source carrier configurations conducted in this paper has allowed us to validate the concept of a new hypothesis test for the detection of a constant speed moving sources under challenging signal-to-noise ratios (from 2 down to 0.8 ). This test, which makes use of Poisson law assumptions, known to govern the generation of a radioactive process, for the estimation of expected mean and variance of a temporal product vector, has proven itself able to ensure a better tradeoff between detection sensitivity and false alarm susceptibility than its previously introduced alternative, based solely on the empirical mean and variance of the product vector Such significant improvement in the sensitivity/false alarm compromise of the detection has been reached without any degradation of the robustness of the test, as its optimization range remains consistent, for a given background, under varying SNR.

Among major limitations to the benchmarked algorithms, two are common to the test disclosed in Coulon et al. and the one introduced in this paper. First, the optimal coverage factor must be set with regards to a previously known value for the recorded background radiation activity. Second, the source has to move with a constant speed, or at least a non-significantly varying one, along the RPM line for the product vector, as hereby calculated, to enable the exploitation of the echo. Our next exploration for improvements will then be orientated toward the softening of this constraint, for instance replacing the condition of constant speed, better suited for pedestrian applications than for the monitoring of a train entering a station, by a condition of speed decreasing according to a constant gradient, The second main limitation only concerns the hypothesis test introduced in this paper, which, by definition, becomes ineffective when the signals registered on the different RPMs diverge from the ones that could be reconstructed using a Poisson law model. Such distortion may for instance arise in the presence of pile-up at the acquisition electronics level, and would, once diagnosed, impose the use of the empirical mean and variance in the detection hypothesis test.

In addition, when inspecting a potential vehicle carrier such as an automobile or a train, the users must address the issue of shielding effect, which was primarily left aside in the assessment scheme described in Section 5: the carrier itself, due to its size, density and effective atomic number, may along its passage shield partially the RPM network from the radiation background. The optimal coverage factor for the detection tests being set with respect to a known and supposedly stable background activity, as the independent detection thresholds are in more conventional approaches, this effect, when non-negligible, systematically affects the performances of the system. A first set of simulations has notably stressed that, for a given attenuation of the recorded background activity, the bias induces a more significant loss in detection sensitivity when the source is placed at the front or at the back of the vehicle. False detections at the end of the passage may also have to be deplored, as the transition from the attenuated background level to the initial one may be mistakenly attributed to the addition of a radioactive source. A refinement of the algorithm hereby disclosed is currently under patent deposition at the CEA LIST, which specifically targets the limitation of this shielding effect.

The detection algorithm described in this article, together with the one presented in Coulon et al. is to be implemented into a prototype system developed in the course of the SECUR-ED European project for urban mass transportation [16]. Each RPM sensor of the network is made of two $10 \times 10 \times 100 \mathrm{~cm}^{3}$ plastic scintillators of EJ-200 type produced by Eljen Technology. A demonstration campaign will be thoroughly conducted to ensure the compliance of both the sensor device and the detection algorithms to the international standards (17) labeled ANSI N42.35.

\section{Acknowledgments}

The authors would like to thank the members of the SECUR-ED consortium for having implicated them in their research.

\section{References}

[1] Chemical, biological, radiological or nuclear (CBRN) detection: a technological overview, NATO Parliamentary Assembly, 2005.

[2] RADOS DnD-Portal, MIRION Technologies Technical file, 2010.

(3) Radiological control of vehicle load, SAPHYMO Technical file 29294835 EN, 2011.

[4] L. Guillot, A. Reboli and A. Dominique, Dispositif et procédé de détection et d'identification en temps réel d'une source radioactive en mouvement, 2008.

[5] S.M. Robinson, S.E. Bender, E.L Flumerlelt, C.A. Lopresti, M.L. Woodring, IEEE, Transactions on Nuclear Science N556 (6) (2009) 3688.

[6] R.B. Vilim, R. Klann, C. Fink, C. Campos and T. Medley, Proceedings of the IEEE Conference on Technology for Homeland Security, 2007, p. 174.

[7] S. Brennan, A. Mielke, D. Torney, IEEE Transactions on Nuclear Science NS52 (3) (2005) 813 .

[8] ].-C Chin, N. Rao, D. Yau, M. Shankar, Y. Hou, J. Hou, S. Srivathsan, S. Iyengar, AMC Transactions on Sensor Networks 7 (3) (2010) 135. 
[9] R. Nemzek, J. Dreicer, D. Torney, T. Warnock, IEEE Transactions on Nuclear Science NS51 (4) (2004) 1693.

[10] D. Stephens, A. Peurrung, IEEE Transactions on Nuclear Science NS51 (5) (2004) 2273.

[11] A. Sundaresan, P.K. Varshney and N.S.V. Rao, Proceedings of the International Conference on Information Fusion, 2007, pp. 1.

[12] N.S.V. Rao, S. Sen, N.J. Prins, D.A. Cooper, R.J. Ledoux, J.B. Costales, K. Kamieniecki, S.E. Korbly, J.K. Thompson, J, Batcheler, R.R. Brooks, C.Q. Wu. Nuclear Instruments and Methods in Physics Research A 784 (2015) 326.

[13] R. Coulon, V. Kondrasovs, S. Normand and M. Bakkali, Commissariat à I'Energie Atomique et aux Energies Alternatives, Procédé de détection de source radioactive en mouvement et dispositif associé, 2014
[14] R. Coulon, V. Kondrasovs, K. Boudergui, S. Normand, IEEE Transactions on Nuclear Science NS61 (4) (2014) 2189.

[15] G. Knoll, Radiation Detection and Measurement, Fourth ed., John Wiley \& Sons, Inc., New York (1999) pp. 94-95.

[16] K. Boudergui, V. Kondrasovs, R. Coulon, G. Corré and S. Normand, New monitoring system to detect a radioactive material in motion, in: Proceedings of the Advancements in Nuclear Instrumentation Measurement Methods and their Applications, ANIMMA, 2013.

[17] American national standard for evaluation and performance of radiation detection portal monitors for use in homeland security, ANSI 42.35, 2006. 